\title{
Survey on Red Tacton Technology for Human Area Network
}

\author{
S. Sibi Chakkaravarthy, K. A.Varun Kumar, G. Arun Karthick, K. Kavitha \\ ${ }^{1,2,3}$ Dept. of CSE Vel Tech University, Chennai \\ ${ }^{4}$ Dept. of CSE Centre for Development of Advance computing,CDAC-Pune
}

\begin{abstract}
Red Tacton was one of the advanced Pervasive technology that are genuinely user-friendly to everyone will require technologies that enablecommunication between people and objects in close proximity. Focusing on the naturalness, inevitability, and sense of security conveyed by touching in everyday life, this article describes human area networking technology that enables communication by touching, which we call RedTacton. Here, the human body acts as a transmission medium supporting IEEE 802.3 half-duplex communication at 10Mbit/s. The key component of the transceiver is an electric-field sensor implemented with an electrooptic crystal and laser light.RedTacton uses the minute electric field generated by human body as medium for transmitting the data.The chips which will be embedded in various devices contain transmitter and receiver built to send and accept data in digital format.In this paper we surveyed the red tactontechnology, working principle of red tacton over human area network, application,protocols for data transmission etc.
\end{abstract}

Keywords: Red Tecton, HAN,

\section{Introduction:}

Today people can communicate anytime, anywhere, and with anyone over a cellular phone network.Moreover, the Internet lets people download immense quantities of data from remotely locatedservers to their home computers. Essentially, these two technologies enable communications betweenterminals located at a distance from each other. Meanwhile, all kinds of electronic devices includingpersonal digital assistants (PDAs), pocket video games, and digital cameras are becoming smaller, sopeople can carry around or even wear various personal information and communication appliances during their everyday activities. However, userfriendly ubiquitous services involve more than just networking between remotely located terminals. Communication between electronic devices on the human body (wearable computers) and ones embedded in our everyday environments, so this has driven extensive research and development on human area networks. Wired connections between electronic devices in human area networks are cumbersome and can easily become entangled. Short-range wireless communication systems such as Bluetooth and wireless local area networks (IEEE $802.11 \mathrm{~b}$, etc.) have some problems. Throughput is reduced by packet collisions in crowded spaces such as meeting rooms and auditoriums filled with people and communication is not secure because signals can be intercepted. The principle drawback of infrared communications (IrDA) is the tight directionality of beams between terminals needed for the system to be effective. The ultimate human area network solution to all these constraints of conventional technologies is "intrabody" communication, in which the human body serves as the transmission medium. In ubiquitous services (which imply communication between electronic devices embedded in the environment in close proximity to people), if we could use the human body itself as a transmission medium, then this would be an ideal way of implementing human area networks because it would solve at a stroke all the problems including throughput reduction, low security, and high network setup costs.

\section{Survey about HAN:}

The concept of intrabody communication, which uses the minute electric field propagated by the human body to transmit information, was first proposed by IBM [1].The communication mechanism has subsequently been evaluated and reported by several research groups around the world. However, all those reported technologies had two limitations:

1) The operating range through the body was limited to a few tens of centimeters and

2) The top communication speed was only $40 \mathrm{kbit} / \mathrm{s}$.

These limitations arise from the use of

an electrical sensor for the receiver. An electrical sensor requires two lines (a signal line and a ground line), whereas in intrabody communication there is essentially only one signal line, i.e., the body itself, which leads to an unbalanced transmission line, so the signal is not transmitted correctly.NTT has had excellent success with an electro-optic sensor combining an electrooptic crystal with laser light and recently reported an application of this sensor for measuring high-frequency electronic devices [2],[3].

The electro-optic sensor has three key features: 
(1) it can measure electric fields from a device under test (DUT) without contacting it, which minimizes measurement disturbance,

(2) ultrawide-band measurement is possible, and

(3) it supports one-point contact measurement that is independent of the ground, which is the most significant feature in the present context. NTT utilized this third feature to fabricatean intrabody communication receiver for its human area networking technology, which is called RedTacton*.

The functional principle of RedTactonis illustrated in Fig. 1. The electric field induced towards the bodyby the transmitter's signal electrode is represented by $E a$. The system requires a ground close to the transmitter signal electrode, so electric field Ebinduced from the body can follow a return path to the transmitter ground. Moreover, since people are usually standing on a floor or the ground, electric field Ecescapes from the body to ground, mainly from the feet.

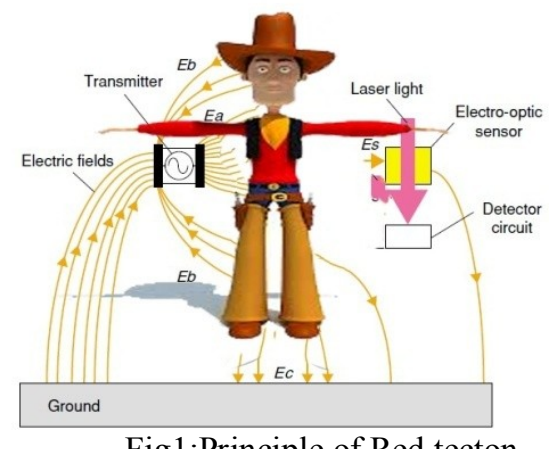

Fig1:Principle of Red tecton

The electric field Esthat reaches the receiver is $E s=E a-(E b+E c)$. Itcouples to the electro-optic crystal and changes the crystal's optical properties. This change is detected by laser light and transformed into digital databy a detector circuit.

\section{Transmission modes:}

1. The Red Tacton transmitter induces a weak electric field on the surface of the body.

2. The Red Tacton receiver senses changes in the weak electric field on the surface of the body caused by the transmitter.

3. It relies on the principle that the optical properties of the electro-optic crystal varies according to the changes in the weak electric field.

4. It detects the changes in the optical properties of an electrooptic crystal using a laser beam and converts the result into an electrical signal in a detector circuit.

\section{Tacton Transceiver:}

The signal from the interface is sent to the data sense circuit and the transmitter circuit. The data sense circuit senses the signal and if the data is present it sends control signal to the transmitter which activates the transmitter circuit. The transmitter circuit varies the electric field on the surface of our body. This change in the electric field is detected by the electro-optic sensor. The output of the electrooptic sensor is given to the detector circuit, which in turn given to the interface of the receiving red tacton device. Thus the Data is transmitted from one device to another device .Here the reliable data transmission is taken place with the own device MAC address.

\section{Safety measures of Red Tacton over Human Body:}

We probed that the effects of Red Tacton technology on human body which is evidently an spectacular issue of the red tacton technology. First as shown in figure2, the transmitting and receiving electrodes of the Red Tacton receiver are completely covered with insulating film, so the body of the person acting as a transmission medium is completely insulated. This makes it not possible for electron particle in current to flow into a person's body from a transceiver. When communication is initiated, displacement current is generated by the electrons in the body because the body is subjected to minute electrical fields change. However such displacement currents are very common everyday occurrences to which we are all subjected. Red Tacton conforms to the "Radio Frequency- Exposure Protection Standard (RCR STD-38)" [6] issued by the association of Radio industries and business (ARIB). The levels produced by Red Tacton are well below the safety limit specified by this standard. 


\section{Application of Red Tacton Technology:}

There are many applications for red tacton in wide area of Technology With the advancement of wireless technology, new applications become possible. The red tacton project is a collaboration of relevant parties to investigate new mobile applications in the healthcare sector,data transmission,communication sectors. As part of this project the Body Area Network (BAN) or Human area network has been developed.

\section{Touch \& Touch:}

Touching, gripping, walking, sitting, steeping and other human movements can be the triggers for unlocking or locking, starting or stopping equipment, or obtaining data. Here data is transmitted through touching the human hand surface in transmitting datas.Performing authentication by gripping the door locks for locking and unlocking.
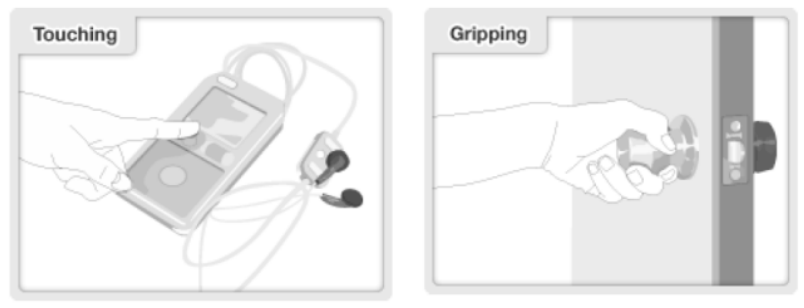

Fig.2:Touch\& Touch

\section{Red Tacton in Biomonitoring systems:}

The main purpose of the BAN is to make it possible for patients who need permanent monitoring to be fully mobile. To fulfill that objective a personal lightweight monitor system is created that is completely customized to the patientsneeds. The BAN is worn by a patient and basically consists of a set of lightweight devices that monitor and wirelessly transmit certain bio signals (vital signs) to a BackEnd System. Healthcare centers can then retrieve this data over a reliable wired connection.

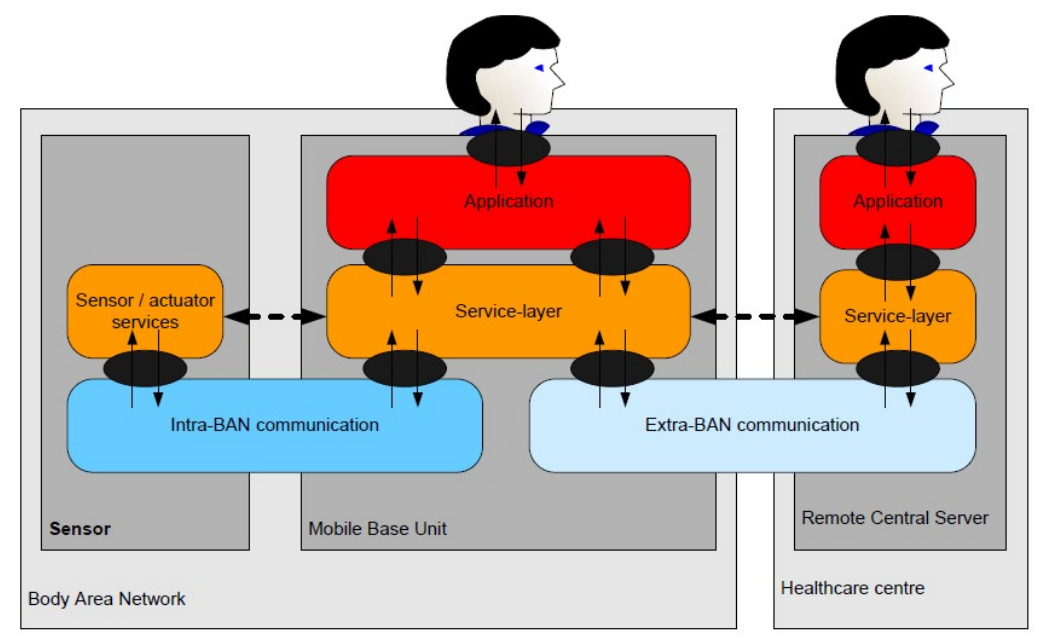

Fig.3:Biomonitoring system $-\mathrm{HAN} / \mathrm{BAN}$

\section{Han in under water communication:}

Red Tacton allows communication in outer space and in water where the speech constraints are very high and thus enables a highly efficient means of expression of speech which is beyond the purvey of human beings.
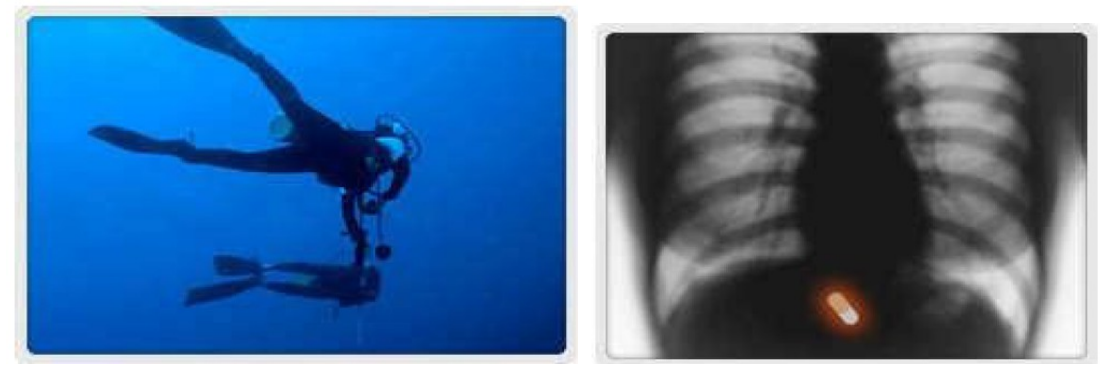

Fig.4:Communication under water and inside body 
Red Tacton in Flight seating,Exclators etc.
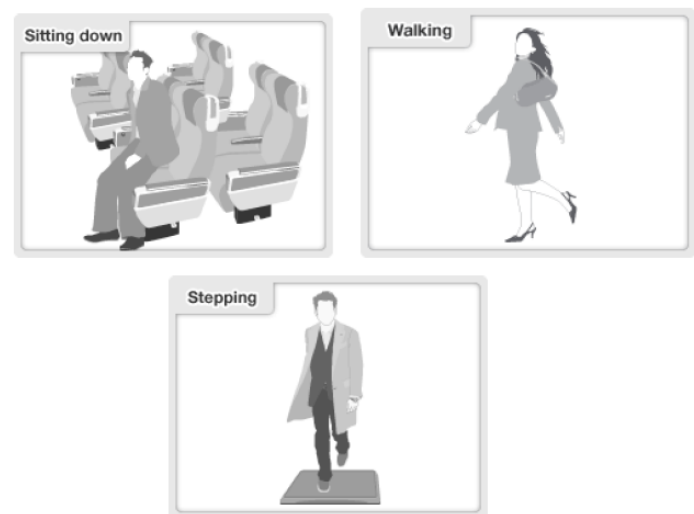

Fig.5:Redtacton device triggered by sitting, Walking,Stepping

Network \&InterNetwork\&Intra Network via Red Tacton(HAN):
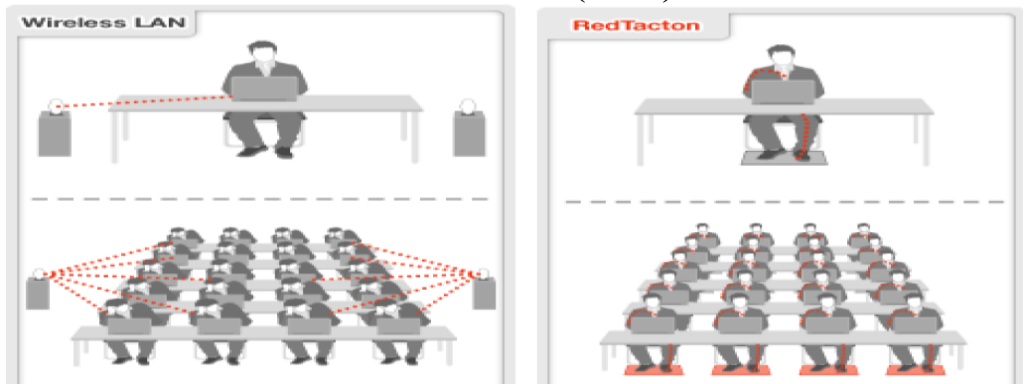

Fig.6:Comparision between WLan\& Red Tacton

\section{Comparision of red Tacton with WPAN,UWB:}
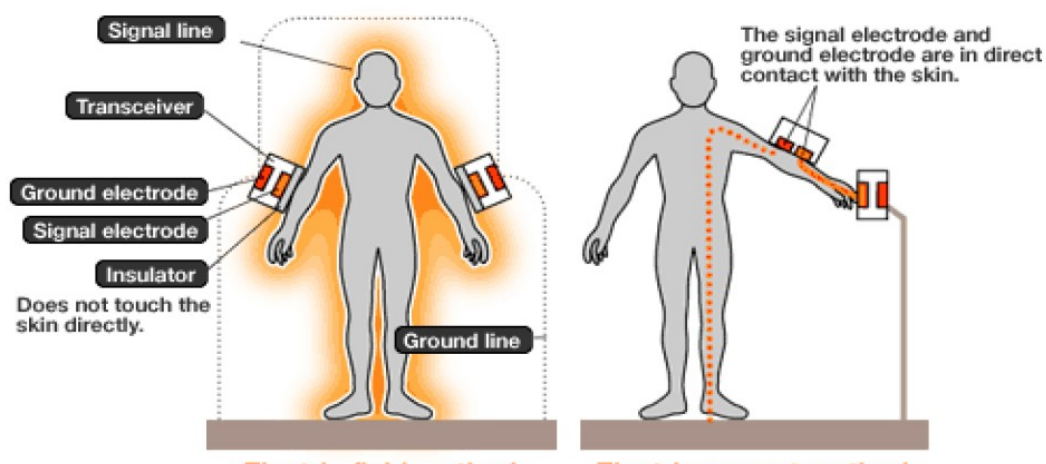

Electric field method

Electric current method

Fig.7:Comparision of Red Tacton with other Human Body Based Communication Channels[4].

*RedTacton does not require the electrode to be in direct contact with the skin.With the electric amperage method, electrode must be in direct contact with the skin.

\section{Comparision of Red Tacton with other networks:}

\begin{tabular}{|l|l|l|c|c|c|}
\hline Protocol & $\begin{array}{l}\text { Range } \\
\mathrm{m}\end{array}$ & $\begin{array}{l}\text { Data } \\
\text { M.Byte }\end{array}$ & $\begin{array}{c}\text { Speed } \\
\text { Mbps }\end{array}$ & Iterationswith Multiple Users & Duplex Data \\
\hline Red Tacton & $10-15$ & 10 & 10 & Good & Poor \\
\hline Bluetooth & Max 100 & $0.5-1$ & $256-1$ & Average & Poor \\
\hline Zigbee & 30 & $0.5-0.8$ & $256-512$ & Average & Poor \\
\hline UWB & $50-60$ & $0.4-0.5$ & 1 & Poor & Better \\
\hline Infra Red & $5-8$ & $0.3-0.5$ & $128-256$ & Poor & Poor \\
\hline Wlan & $6-7 \mathrm{~km}$ & unlimited & unlimited & Excellent & Excellent \\
\hline
\end{tabular}




\section{Future Enhancement:}

longer-term plans include developing a mass-market transceiver interface supporting PDAs and notebook computers while continuing efforts to reduce the size and power consumption of the transceiver to enhance its portability.

\section{Conclusion:}

Red tacton technology is one of the best adaptive range of communication network for wireless pervasive as well ubiquitous environment,It provides various application with less fault tolerance and it can be supportive for mobile devices also. The evolution of Red Tacton technology is a boundless success, which likely to be battered for pervasive world in future.Finally I conclude that Future is full of Pervasive Environment which is fully represented by Red Tacton technology as the communication path.

\section{References}

[1] T.G.Zimmerman, "Personal Area Networks: Near-field intrabody communication,” IBM systems journal, Vol. 35, Nos. 3\&4, pp.609-617, 1996.

[2] T.Nagatsuma and M.Shinagawa, "Photonic measurement technologies for high frequency electronics," NTT REVIEW, Vol.14, No.6.pp. 12-24, 2002.

[3] M.Shinagawa, "Development of Electro-optic sensors for Intra-body Communication,” NTT Technical Review, Vol. 2, No. 2, pp. 6-11, 2004.

[4] M.Shinagawa, M. Fukumoto, K. Ochiai, and H. kyuragi, "A near-field-sensing transceiver for intra-body communication based on the electro-optic effect,” IEEE Trans.IM, Vol.53,No.6, pp. 1533-1538,2004.

[5] M. Mizoguchi, T.Okimura, and A.Matsuda, "Comprehensive commercialization Functions," NTT Technical Review, Vol.3, No. 5, pp. 12-16, 2005.

[6] Scribd (2010) "Human Area Networks- Red Tacton". Available: http://www.scribd.com/doc/55240946/RED-TACTON-REPORT.

[7] Anil K. Jain, Patrick Flynn, and Arun Abraham Ross- Handbook of biometrics;FirstEdition;Springer, 2007.

[8] Zheru Chi, Hong Yan, and TuânPh?m-Fuzzy algorithms: with applications to image processing and pattern recognition;FirstEdition;World Scientific, 1996.

[9] Wikipedia.org

[10] Sang-KyunIm, Hyung-Man Park, Young-Woo Kim, Sang-Chan Han, Soo-Won Kim, Chul-Hee Kang and Chang-Kyung Chung, "Biometric Identification System by Extracting Hand Vein Patterns", Journal of the Korean Physical Society,Vol.38, No.3,March 2001.

[11] Sang KyunIm, Hwan Soo Choi, and Soo-Won Kim,"A Direction- Based Vascular Pattern Extraction Algorithm for Hand Vascular Pattern Verification",ETRI Journal, Vol.25, No.2, April 2003.

[12] Debnath Bhattacharyya, Poulami Das, Tai-hoonKim,and Samir Kumar Bandyopadhyay,"Vascular Pattern Analysis towards Pervasive Palm Vein Authentication",Journal of Universal Computer Science,Vol.15,No.5,March 2009.

[13] KavehPahlavan, and Prashant Krishnamurthy-Networking Fundamentals: Wide, Local and Personal Area Communications; FirstEdition;John Wiley and Sons, 2009. 